\title{
Efficacy and Acceptability of Office Hysteroscopy before Assisted Reproductive Technology
}

\author{
Kunur Shah ${ }^{1}$, Vineet V Mishra ${ }^{2}$, Rohina Aggarwal ${ }^{3}$, Sumesh Choudhary ${ }^{4}$, Smit B Solanki ${ }^{5}$
}

\begin{abstract}
Introduction: Hysteroscopic technique of diagnosis and treatment of intrauterine pathology without anesthesia has gained popularity over the last few years. Intrauterine pathologies are found to be present in a significant number of infertile patients. Structural abnormalities of the endometrial cavity may affect the reproductive outcome adversely, by interfering with implantation or causing spontaneous abortion. Therefore, exclusion of any intrauterine pathology becomes an important step in infertility work-up prior to IVF.

Objectives: This study was aimed to estimate the safety, efficacy, and patients' acceptability of office hysteroscopy $(\mathrm{OH})$ for evaluating the uterine cavity in patients of infertility planned for in vitro fertilization.

Materials and methods: This is a prospective observational study enrolling 147 women. All women who were planned for IVF and underwent hysteroscopy were included in the study, and their data were analyzed. Office hysteroscopy was done without anesthesia, and their pain was rated on a Numerical Pain Rating Scale.

Results: Pain was evaluated using a 10-cm visual analog scale. Statistical analysis was performed using SPSS version 20 . Mean age of total patients is $33.51 \pm 1.89$ years. The mean pain score immediately after the procedure was $2.84 \pm 1.26$, and after 15 minutes, it was $0.95 \pm 1.20$. Majority of the patients tolerated the procedure well.

Conclusion: The possibility of doing office hysteroscopy on outpatient basis without anesthesia and accuracy in diagnosing intrauterine abnormalities makes it a gold standard procedure.

Keywords: Gold standard, Numerical pain rating scale, Office hysteroscopy.

Journal of South Asian Federation of Obstetrics and Gynaecology (2021): 10.5005/jp-journals-10006-1895
\end{abstract}

\section{INTRODUCTION}

Uterine and cervical pathologies have a significant impact on success of assisted reproductive technology (ART). In previous studies, $25 \%$ of infertile women were identified having intrauterine pathologies. ${ }^{1}$ Saravelos in his critical analysis of studies from 1950-2007 identified that $6.7 \%$ of the general population, $7.3 \%$ of the patients with infertility, and $16.7 \%$ of women having recurrent pregnancy loss were found to have congenital uterine anomalies. ${ }^{2}$ Intrauterine pathologies may impair fertility outcome by implantation failure or by predisposing to spontaneous abortion ${ }^{3}$ so the evaluation of uterine cavity before starting IVF protocol is of utmost importance. There are various modalities by which intrauterine abnormalities can be visualized like transvaginal sonography (TVS), three-dimensional ultrasonography (3D TVS), hysterosalpingography (HSG), saline infusion sonography (SIS), sonohysterography, and hysteroscopy.

Every diagnostic modality has its own benefits and shortcomings. HSG has high sensitivity (98\%), but its use is limited due to lower specificity (34.9\%). ${ }^{4}$ As compared to HSG, TVS has higher sensitivity (100\%) and specificity (96.3\%). ${ }^{5}$ Alexis et al. demonstrated no statistically significant difference in fertility treatment outcome in patients who have undergone sonohysterography as compared to those who have opted for other modalities of uterine cavity evaluation. ${ }^{6}$

Even though the routine use of hysteroscopy in evaluating all infertility patients is questioned by many authors, hysteroscopy is still believed to be the "Gold Standard" procedure for the same. Miniature hysteroscope allows us to perform hysteroscopy in office setting along with minor operative interventions. ${ }^{7}$

\footnotetext{
${ }^{1-5}$ Department of Obstetrics and Gynecology, Institute of Kidney Diseases and Research Centre, Ahmedabad, Gujarat, India

Corresponding Author: Kunur Shah, Department of Obstetrics and Gynecology, Institute of Kidney Diseases and Research Centre, Ahmedabad, Gujarat, India, e-mail: drkunurshah22@yahoo.co.in

How to cite this article: Shah K, Mishra VV, Aggarwal R, et al. Efficacy and Acceptability of Office Hysteroscopy before Assisted Reproductive Technology. J South Asian Feder Obst Gynae 2021;13(3):142-145.

Source of support: Nil

Conflict of interest: None
}

Different centers follow different policies regarding employment of office hysteroscopy before ART like (i) hysteroscopy to be performed upon suspecting intrauterine pathology at HSG, transvaginal ultrasonography, or SIS; (ii) cases of ART cycle(s) failure although normal diagnostic modalities; (iii) hysteroscopy done routinely before all IVF cycles. ${ }^{8}$ In our center, being governmentfunded trust hospital, we have adopted the third strategy that routine hysteroscopy is performed prior to starting IVF protocol in all patients.

\section{Materials and Methods}

The study design is a prospective observational study conducted on 147 women with infertility who were scheduled for IVF/ Intracytoplasmic Sperm Injection (ICSI) treatment in the IVF unit at Institute of Kidney Diseases and Research Centre, Ahmedabad, India, from January 2020 to August 2020. Approval of the 
Institutional Ethical Committee (IEC) was taken prior to initiating the study and formulating a study protocol. Our objectives were (a) to evaluate the efficacy of outpatient hysteroscopy in identifying intrauterine pathologies and (b) to evaluate the pain perceived by patients, which in turn determines the overall acceptability of the procedure. Following were the inclusion and exclusion criteria.

\section{Inclusion Criteria}

- Planned for IVF/ICSI

- Recurrent abortions

- Post-abortal amenorrhea

- Foreign body in the uterus

\section{Exclusion Criteria}

- Medical disorders-cardiovascular disease, Chronic Obstructive Pulmonary Diseases (COPD), bleeding disorder

- Gross diagnosed pelvic pathology

- Infection

Patients were recruited according to the inclusion and exclusion criteria mentioned above. After explaining the process of office hysteroscopy, all the patients and the attendees gave informed and written consent.

Clinical history of all patients was evaluated with reference to duration and causes of infertility, previous diagnostic tests performed, and past medical treatment received. Complete clinical examination was performed before the procedure. Both 3D TVS and office hysteroscopy were scheduled in postmenstrual period in the early-mid follicular phase of the same menstrual cycle, before starting the IVF/ICSI treatment. All patients received injection atropine $0.6 \mathrm{mg}$, injection hyoscine $20 \mathrm{mg}$, tablet misoprostol $400 \mu \mathrm{g}$ per vaginum at 6 a.m., and prophylactic antibiotic tablet amoxicillin with clavulanic acid $625 \mathrm{mg}$ single dose 1 hour prior to the procedure.

Office hysteroscopy was done without any anesthesia using a rigid continuous flow hysteroscope of $2.9 \mathrm{~mm}$ in diameter with $3.8 \mathrm{~mm}$ sheath and 12 degree fiber optic scope (Karl Storz Endoscopy, Germany) with no-touch technique. Uterine cavity distention was achieved with normal saline with a distension pressure of 80-100 mm Hg. Vaginoscopy was performed. After identifying the cervix and external os, hysteroscope was slowly negotiated in the cervical canal and subsequently into the uterine cavity. Stepwise observation of the uterine cavity was performed, including panoramic view anterior, posterior, lateral walls, fundus, and bilateral ostia. Any pathology observed was noted with context to size, shape, and location. Cervical canal was visualized carefully for any abnormalities while withdrawing the scope. Complications or difficulties that occurred during the procedure were recorded. Average duration of the procedure was 6-8 minutes.

\section{Numerical Pain Rating Scale (NPRS)}

Assessment of pain perception was done by a Numerical Pain Rating Scale (NPRS, 0-10) (Fig. 1). The NPRS is a segmented numeric version of the visual analog scale (VAS) in which a respondent selects a whole number (0-10 integers) that best reflects the intensity of her pain. ${ }^{9}$ It is represented as a horizontal bar or line. Severe pain perceived during the procedure (NPRS $\geq 7$ ) or moderate to severe at discharge (NPRS $\geq 4$ ) were considered unacceptable. ${ }^{10}$

\section{Statistical Methods}

Analysis was done by SPSS version 20. Data were expressed as mean \pm standard deviation and percentage where applicable.

\section{Results}

A total of 147 women were enrolled in the study. Mean age of the patients was $33.51 \pm 1.89$ years (Table 1 ). Majority of women had primary infertility (88.63\%).

The main indication of office hysteroscopy was pre-IVF evaluation of uterine cavity; $70.74 \%$ (104) had normal hysteroscopy. The prevalence of uterine cavity abnormalities was $29.25 \%$ (43) as detected by hysteroscopy in our study population. Various abnormal clinical findings were observed, including endometrial polyp, cervical polyp, submucous myoma, uterine septum, intrauterine adhesions etc. Hypertrophic/polypoidal endometrium was the most common abnormality reported followed by endometrial polyp and unicornuate uterus (Table 2).

Out of 43 clinical abnormalities identified, nine patients required further intervention in operation theater under anesthesia like hysteroscopic polypectomy, submucous myomectomy, septal resection, hysteroscopic adhesiolysis. Two cases of cervical polypectomy, five cases of endometrial polypectomy, and four cases of minor adhesiolysis were performed in office setting without anesthesia. Cervical stenosis was identified in two patients, which was managed by sounding followed by gentle negotiation of hysteroscope, without the need of anesthesia.

Most of the women, immediately after the procedure as well as 15 minutes after the procedure, perceived a minimal pain, with a pain score of $0-3$. Table 3 shows the relation of parity with pain experienced by the patient, respectively; $81.63 \%$ of women perceived mild pain (pain score: $0-3$ ) immediately after the procedure (Table 4). The pain factor further diminished in women

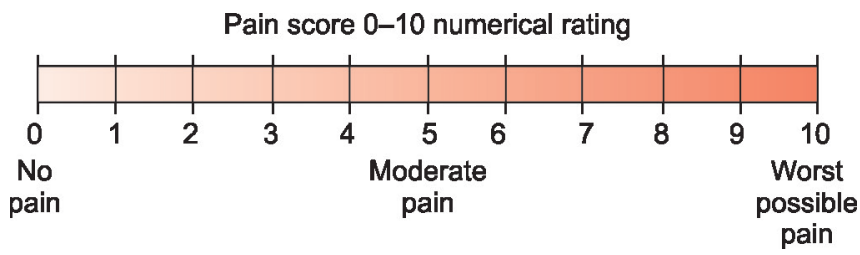

Fig. 1: Numerical Pain Rating Scale (NPRS 0-10)

Table 1: Demographic characteristics

\begin{tabular}{lc}
\hline Characteristic & Values $(n=147)$ \\
\hline Age (years) & $33.51 \pm 1.89$ \\
Type of infertility & \\
Primary infertility & $120(88.63 \%)$ \\
Secondary infertility & $27(18.36 \%)$ \\
Duration of infertility (years) & $5.16 \pm 3.22$ \\
Etiology of infertility & \\
Male factor & $37(25.17 \%)$ \\
Ovarian factor & $41(27.89 \%)$ \\
Tubal/peritoneal factor & $29(19.72 \%)$ \\
Combined factors & $19(12.92 \%)$ \\
Unexplained & $21(14.28 \%)$ \\
\hline
\end{tabular}


Table 2: Hysteroscopic findings

\begin{tabular}{ll}
\hline Cervical pathology & 2 \\
Adhesion & 1 \\
Cervical fibroid & 2 \\
Cervical polyp & 1 \\
Cervical fibrous band & 2 \\
Cervical stenosis & \\
Uterine cavity abnormality & 4 \\
Unicornuate & 1 \\
Bicornuate & 1 \\
Adhesion & 1 \\
Complete septum & 2 \\
Subseptate uterus & 8 \\
Endometrial polyp & 1 \\
Previous scars of perforation & 1 \\
Asherman syndrome & 2 \\
Submucous myoma & 2 \\
Endometrial pathology & 10 \\
Hypertrophic/polypoidal endometrium & 2 \\
Atrophic endometrium & \\
Subendometrial hemorrhagic spot & \\
\hline
\end{tabular}

Table 3: Parity classification and pain score

\begin{tabular}{lll}
\hline Parity & Pain score & \\
\hline & Immediate & After 15 minutes \\
Multiparity & $2.82 \pm 1.25$ & $0.92 \pm 0.14$ \\
Nulliparity & $2.93 \pm 1.30$ & $1.07 \pm 1.44$ \\
\hline
\end{tabular}

Table 4: Pain perception immediately after the procedure

\begin{tabular}{lcc}
\hline Pain score & No. of patients (\%) & Mean \pm SD \\
\hline $0-1$ & $11(7.48 \%)$ & $0.91 \pm 0.30$ \\
$2-3$ & $109(74.15 \%)$ & $2.53 \pm 0.50$ \\
$4-5$ & $20(13.61 \%)$ & $4.20 \pm 0.41$ \\
$6-7$ & $05(3.40 \%)$ & $6.20 \pm 0.45$ \\
$8-10$ & $02(1.36 \%)$ & $8 \pm 0$ \\
\hline
\end{tabular}

15 minutes after the procedure, and $72.79 \%$ women experienced a pain score of $0-1$ (Table 5 ). The mean pain score immediately after the procedure was $2.84 \pm 1.26$, and after 15 minutes, it was $0.95 \pm 1.20$.

In our study, there were no complications recorded with office hysteroscopy, except for the fact that there was failed hysteroscopy in one patient due to cervical stenosis, abnormal uterine position, and noncompliance. We did not observe any perforation, hemorrhagic, or metabolic complications due to office hysteroscopy.

The mean volume of distension fluid (NS) used in the patients was $99.87 \pm 3.85 \mathrm{~mL}$. Two patients (1.36\%) experienced severe pain (pain score 8 ) and required injectable analgesics; $98.63 \%$ of patients
Table 5: Pain perception after 15 minutes of the procedure

\begin{tabular}{lcc}
\hline Pain score & No. of patients (\%) & Mean \pm SD \\
\hline $0-1$ & $107(72.79 \%)$ & $0.36 \pm 0.48$ \\
$2-3$ & $35(23.81 \%)$ & $2.14 \pm 0.36$ \\
$4-5$ & $03(2.04 \%)$ & $4.67 \pm 0.58$ \\
$6-7$ & $02(1.36 \%)$ & $6 \pm 0$ \\
$8-10$ & - & - \\
\hline
\end{tabular}

very well accepted hysteroscopy; only two patients (1.36\%) desired to undergo this procedure under anesthesia.

\section{Discussion}

Intrauterine pathologies like endometrial polyps, submucous myomas, intrauterine adhesions, and septa may adversely affect the fertility outcome of ART procedures. ${ }^{11}$ Normal uterine cavity is essential prerequisite for successful IVF outcome. That is why pre-evaluation of endometrial cavity is necessary for all the patients before starting IVF cycle. In Indian scenario, IVF treatment seems beyond the affordable capacity for a segment of infertile families. By performing hysteroscopy in office setting, we can reduce the significant cost and time as well as exposure of anesthetic hazards.

Nowadays, 3D TVS has gained a significant popularity in gynecological practice. It improves diagnostic accuracy in those complicated cases where conventional 2D scanning and other diagnostic modalities have ended up in diagnostic dilemma. Although 3D TVS is easily available and noninvasive modality to evaluate the endometrial cavity and uterus, its accuracy, sensitivity, and specificity are lower than those of office hysteroscopy. ${ }^{12,13}$

Hysteroscopy has been regarded as the gold standard and definitive procedure for the exploration and evaluation of uterine cavity. Safety and simplicity of the procedure allow it to be performed in office setting. Office hysteroscopy provides good diagnostic accuracy at a lower cost with good patient's acceptability as compared to conventional hysteroscopy. ${ }^{14}$

Angioli et al. identified the role of music for lowering pain perception in operative hysteroscopic procedures in office settings as compared with procedures performed without music. ${ }^{15}$ In another Indian study, it was stated that women in outpatient hysteroscopy units experience higher levels of anxiety as compared to other patients in gynecology care. Carta et al. deduced increased waiting time and increased age to be associated with more pain, but no increase in anxiety, ${ }^{16}$ while Kokanali et al. found that the pain experienced by study participants during and immediately after office hysteroscopy was negatively affected by pre-procedure anxiety level and reducing waiting time may decrease the anxiety. ${ }^{17}$

\section{Conclusion}

Uterine cavity abnormalities are considered to have a negative impact on the embryo implantation rates in IVF. Among the various available modalities to assess the uterine cavity prior to IVF, office hysteroscopy should be considered as the primary modality in low-resource country like India. Possibility of doing it on outpatient basis without anesthesia, accuracy in diagnosing intrauterine abnormalities and well tolerated and accepted by patients makes office hysteroscopy a gold standard procedure this era. 


\section{References}

1. Levi Setti PE, Colombo GV, Savasi V, et al. Implantation failure in assisted reproduction technology and a critical approach to treatment. Ann N Y Acad Sci 2004;1034:184-199. DOI: 10.1196/ annals.1335.021.

2. Saravelos S, Cocksedge K, Li T. Prevalence and diagnosis of congenital uterine anomalies in women with reproductive failure: a critical appraisal. Hum Reprod Update 2008;14(5):415-429. DOI: 10.1093/ humupd/dmn018.

3. El-Mazny A, Abou-Salem N, El-Sherbiny W. Outpatient hysteroscopy: a routine investigation before assisted reproductive techniques? Fertil Steril 2011;95(1):272-276. DOI: 10.1016/j. fertnstert.2010.06.033.

4. Preutthipan S, Linasmita V. A prospective comparative study between hysterosalpingography and hysteroscopy in the detection of intrauterine pathology in patients with infertility. J Obstet Gynaecol Res 2003;29(1):33-37. DOI: 10.1046/j.13418076.2003.00068.x.

5. Shalev J, Meizner I, Bar-Hava I, et al. Predictive value of transvaginal sonography performed before routine diagnostic hysteroscopy for evaluation of infertility. Fertil Steril 2000;73(2):412-417. DOI: 10.1016/ s0015-0282(99)00533-6.

6. Kim AH, McKay H, Keltz MD. Sonohysterographic screening before in vitro fertilization, Fertil Steril 1998;69(5):841-844. DOI: 10.1016/ s0015-0282(98)00045-4.

7. Bettocchi S, Ceci O, Nappi L, et al. Operative office hysteroscopy without anesthesia: analysis of 4863 cases performed with mechanical instruments. J Am Assoc Gynecol Laparosc 2004;11(1):5961. DOI: 10.1016/s1074-3804(05)60012-6.

8. Bozdag G, Aksan G, Esinler I, et al. What is the role of office hysteroscopy in women with failed IVF cycles? RBM Online 2008;17(3):410-415. DOI: 10.1016/s1472-6483(10)60226-x.
9. Diniz DB, DepesDde B, Pereira AM, et al. Pain evaluation in office hysteroscopy: comparison of two techniques. Rev Bras Ginecol Obstet 2010;32(1):26-32. DOI: 10.1590/s0100-72032010000100005.

10. Fonseca MF, Sessa FV, Resende JAD, et al. Identifying predictors of unacceptable pain at office hysteroscopy. J Minim Invasive Gynecol 2014;21(4):586-591. DOI: 10.1016/j.jmig.2013.12.118.

11. Ahmad G, O'Flynn H, Attarbashi S, et al. Pain relief for outpatient hysteroscopy. Cochrane Database Syst Rev 2010;CD007710. DOI: 10.1002/14651858.CD007710.pub2.

12. Vinagre C, Mairos J, Di Martino, P. Hysteroscopic anesthesia: a new method of anesthesia in ambulatory hysteroscopy. Acta Obstet Gynecol Port 2013;7(4):3. http://www.fspog.com/pt/revistas/1-18-28/

13. Skensved $\mathrm{H}$. Hysteroscopically guided intramyometrial local anaesthesia - the focal local - for resection of endometrial polyps by vaginoscopic microhysteroscopy in postmenopausal women. J Minim Invasive Gynecol 2013;20(6):1. DOI: 10.1016/j. jmig.2013.08.373.

14. Paulo AA, Solheiro $M H$, Paulo $C O$. Is pain better tolerated with mini-hysteroscopy than with conventional device? A systematic review and meta-analysis: Hysteroscopy scope size and pain. Arch Gynecol Obstet 2015;292(5):987-994. DOI: 10.1007/s00404-0153731-0.

15. Angioli $\mathrm{R}$, De CiccoNardone $\mathrm{C}$, Plotti F, et al. Use of music to reduce anxiety during office hysteroscopy: prospective randomized trial. J Minim Invasive Gynecol 2014;21(3):454-459. DOI: 10.1016/j. jmig.2013.07.020.

16. Carta G, Palermo P, Marinangeli F, et al. Waiting time and pain during office hysteroscopy. J Minim Invasive Gynecol 2012;19(3):360-364. DOI: 10.1016/j.jmig.2012.01.017.

17. Kokanali MK, Cavkaytar S, Guzel Al, et al. Impact of preprocedural anxiety levels on pain perception in patients undergoing office hysteroscopy. J Chin Med Assoc 2014;77(9):477-481. DOI: 10.1016/j. jcma.2014.07.004. 